\title{
More and more radio pulsars: status of recent surveys and perspectives
}

\section{Andrea Possenti*}

INAF-Osservatorio Astronomico di Cagliari

E-mail: possenti@ca.astro.it

Forty years after the discovery of the first pulsar, radio pulsar science is still a very active and flourishing branch of radio astronomy. It is strongly favoured by the rapid growth of the population of known objects, providing the statistical basis from which a number of very interesting pulsars regularly emerges. Here I give a summary of the available pulsar sample and highlight the role of the most successful recent pulsar search experiments in populating the pulsar catalogs. A summary about the ongoing and planned experiments is also included, discussing the perspectives to unveil new objects in the Galactic field, in the Globular Clusters and in close-by galaxies. We also describe how the interplay between the chosen experimental parameters leads to the search of a specific subset of the pulsar populations.

From planets to dark energy: the modern radio universe October 1-5 2007

University of Manchester, Manchester, UK

\footnotetext{
* Speaker.
} 


\section{The trade off in the choice of the survey parameters}

The radiometer equation provides us with a formula for estimating the minimum mean flux density $S_{\min }$ of a detectable pulsar (see i.e [1]):

$$
S_{\text {min }} \simeq \sigma_{n} \frac{T_{\text {sys }}+T_{\text {sky }}}{G \sqrt{N_{p} \Delta t \Delta v_{M H Z}}} \sqrt{\frac{W_{e}}{P-W_{e}}} ; \quad W_{e}=\sqrt{W^{2}+(\beta \delta t)^{2}+\left(\delta t_{D M}\right)^{2}+\left(\delta t_{\text {scatt }}\right)^{2}}
$$

where $\sigma_{n}$ is the adopted minimum signal-to-noise ratio of a reliable pulsar signal; $T_{\text {sky }}$ the sky background temperature in $\mathrm{K} ; T_{s y s}$ the system noise temperature in $\mathrm{K} ; G$ the antenna gain in $\mathrm{K} / \mathrm{Jy} ; N_{p}$ the number of polarizations; $\Delta t$ the integration time of a single observation in seconds; $\Delta v_{M H Z}$ is the observing bandwidth in $\mathrm{MHz} ; P$ is the source rotational period; $W_{e}$ is the effective pulse width; $W$ is the intrinsic pulse width; $\delta t$ is the sampling time in seconds; $\beta$ a parameter accounting for post-detection time constant and anti-aliasing filter; $\delta v_{M H Z}$ the width of a single frequency channel in $\mathrm{MHz} ; \delta t_{D M}$ the smearing in a single channel due to the dispersion of the signal in the interstellar medium and $\delta t_{\text {scatt }}$ the broadening of an impulsive signal due to scattering in the ISM.

Due to economical, technical and computational limits, any pulsar search experiment can probe only a selected volume of the pulsar parameter space. In the following, we describe how the interplay between different experimental parameters, together with the choice of their values, lead to the search of different pulsar populations.

The best options for minimizing $S_{\min }$ would be a very large total bandwidth $\Delta v_{M H Z}$; an extremely long integration time $\Delta t$ and a very fast sampling rate $1 / \delta t$, for selected values of the remaining parameters. However a good sensitivity for the detection of distant pulsating sources can be secured only by splitting the signal into an adequate number of frequency channels, $\delta v_{M H Z}=$ $\Delta v_{M H Z} /($ number of channels). This can be achieved using hardware devices or suitable software (see e.g. [2]), but of course, the greater the number of channels, the larger the amount of data and the longer the time for its processing. The same computational issues arise when adopting very short sampling time. Due to the Doppler smearing of the signal, long integration times also make very difficult the search for pulsars in close orbits. As to the selection of the best observing frequency $v$, one must take into account various factors, namely: (i) pulsar spectral shape $\propto v^{-\alpha}$ with average $\alpha \sim 1.7$; (ii) galactic background spectrum $\propto v^{-2.7}$; (iii) interstellar scattering $\propto v^{-4.4}$; (iv) interstellar dispersion $\propto \delta v v^{-3}$; (v) telescope beam $\propto v^{-1}$.

In summary high frequencies (typical values $\sim 1400-2300 \mathrm{MHz}$, are selected for surveys in the galactic plane or for targeted searches of SuperNova Remnant cores: they are much less sensitive to the interstellar scattering and need a moderate frequency resolution to remove dispersion. In particular high frequency surveys in the galactic plane are ideal for searching young pulsars $\left(\lesssim 10^{6} \mathrm{yr}\right)$, whose typical progenitors are massive stars born in the disk. Moreover, due to their strong magnetic fields, young pulsars evolve rapidly implying a low number density and a large average distance, thus favoring high observing frequencies. The searches for millisecond pulsars in globular clusters at $\mathrm{DM} \gtrsim 100 \mathrm{pc} \mathrm{cm}^{-3}$ are another suitable target for high frequency experiments. In these stellar systems the bulk of the observed pulsar population underwent a phase of re-acceleration due to mass transfer in binaries; so we expect short rotational periods, whose detection requests the scattering smearing of the pulse to be kept negligible.

On the contrary, low frequencies (typically $300-400 \mathrm{MHz}$ ) are preferred for surveys away from the galactic plane: they take advantage from the wider telescope beams (thus reducing the 
needed number of pointings), the steep pulsar spectra, the lower $T_{s k y}$ and the negligible scattering. Thus, low frequency surveys represent a good option for investigating the low-luminosity tail of the pulsars in the neighborhood of the Sun, where a population of relatively old objects prevails.

\section{Pulsar Census}

As of Jan 2008, the combination of the ATNF catalog [http://www.atnf.csiro.au/research/pulsar/psrcat/] with the catalog for pulsars in Globular Cluster (GC) ([http://www.naic.edu/ pfreire/GCpsr.html], maintained by Freire) gives a total of 1792 radio pulsars, 151 of which are in binaries. The pulsar's companion is another neutron star in at least 8 (probably 9) cases, whereas only one system comprising two active pulsars is known. Focusing on the pulsars in the Galactic field and in the Magellanic Clouds, 80 are young pulsars (with a spin down age $P /(2 \dot{P})<100 \mathrm{kyr}$ ), whereas 79 (59 in binaries and 20 isolated) are mildly or fully recycled pulsars (assumed to be those with $P \lesssim 100$ ms and surface magnetic field $B_{s} \lesssim 10^{10} \mathrm{G}$ ). As to the 137 pulsars detected so far in $25 \mathrm{GCs}, 57$ are isolated, 70 are in binary systems, whereas the binarity of 10 sources is not assessed yet. About other 50 pulsars [Burgay 2007, Keith 2007, Lorimer 2007, Ransom 2007, private communications] have been discovered in recent months and did not enter the ATNF catalog yet. In addition, 11 Rotating Radio Transients (RRATs, [3]) and 2 Anomalous X-ray pulsars (AXPs, [4]) are known to emit radio pulses, although their link with the population of the radio pulsars is under debate yet.

The two most successful pulsar search experiments of the last decade have been the Parkes Multibeam Pulsar Surveys (PMPSs) and the Green Bank Globular Clusters (GBGC) survey.

All the PMPSs exploited the 13 beam receiver at the 64-m dish at Parkes. That allowed to cover the Galactic disk and the surrounding areas $(\simeq 21000$ pointings) while adopting relatively long integration times: 35 minutes (with $\delta t=250 \mu \mathrm{s}$ ) for the the Galactic plane and 4.5 minutes (with $\delta t=125 \mu \mathrm{s}$ ) outside the Galactic disk. This fact, as well as the large useful bandwidth (288 MHz centered at $1374 \mathrm{MHz}$, split in 96 frequency channels) and the low noise of the receiver $\left(T_{\text {sys }} \simeq 23 \mathrm{~K}\right.$ ) allowed it to reach a sensitivity much better than any previous large scale search of the same areas, with a particular improvement for distant sources located in the Galactic plane. Between 1997 and 2007, the combinations of all these experiments (PM-Survey, e.g. [5], Swinburne Surveys, e.g. [6], PH-Survey [7]) resulted in the discovery of 857 pulsars, thus doubling the total pulsar sample. Among them, there are 35 recycled pulsars (according to the aforementioned conditions for selecting them) and some tens of other very interesting objects. The apex of this large observational effort has certainly been the discovery of the first double pulsar ever [8][9].

The GBGC looked at the GCs using a single beam low-noise receiver centered at about 1950 MHz. The signal was sampled every $\delta t \sim 82 \mu$ s and split in $7680.78 \mathrm{MHz}$ wide channels. The wide useful (i.e. relatively clean from interferences) bandwidth $(\Delta v \sim 600 \mathrm{MHz})$ and the long adopted integration time (typically 4-8 hrs per cluster) allowed to fully exploit the large collecting area of the $\sim 100 \times 110-\mathrm{m}^{2}$ GBT radio telescope, resulting in unprecedentedly small sensitivity limits for this kind of searches. The higher frequency (with respect to the previous surveys at 1400 $\mathrm{MHz}$ ) also reduced the smearing of the signal due to dispersion and scattering and/or the absorption due to the possible eclipses of the radio pulsar signal in a close binary. Finally, the limited number of pointings (of order hundreds) with respect to other large scale surveys allowed to investigate the data using the best codes (highly demanding in term of computational effort) for searching for short 
orbital period and rapidly rotating pulsars. As a result, more than 60 recycled pulsars in Globular clusters have been discovered so far, 32 of which in the cluster Terzan 5 (e.g. [10]). Many exciting objects entered the catalog, such as the fastest spinning pulsar (PSR J1748-2446ad, $P=1.396 \mathrm{~ms}$, [11]) and a binary pulsar (PSR J1748-2021B in NGC 6440, [12]) whose total system mass might imply the existence of a neutron star heavier than about $2.5 \mathrm{M}_{\odot}$.

\section{The present and the future of the pulsar searches}

Despite the number of known pulsars which is approaching 2000, important scientific results are expected from a further increase of the sample: e.g. (i) some pulsars are excellent clocks, leading to unique experiments in astrophysics and fundamental physics: gravity theories, nuclear matter, plasma physics; (ii) the availability of many tens of very stable pulsar clocks (vs the handful of objects known so far) will significantly improve the sensitivity of any pulsar timing array; (iii) rare and especially interesting objects are likely to be found only by increasing the total number of known sources; (iv) pulsars are excellent probes of the interstellar medium, hence a larger pulsar sample will lead to a better knowledge of the distribution of ionized matter and magnetic fields in the Galaxy; (v) the discovery of many other pulsars will promote a better understanding of the Galactic distribution and birthrate of pulsars, of binary and stellar evolution, of their relationship to other objects such as supernova remnants, and of the physics of the radio and high energy emission; (vi) the recycled pulsars in a Globular Cluster are powerful and sometimes unique tools for investigating the stellar and the ionized gas content, the dynamics, and the potential well of the cluster. Moreover, given the very high stellar density in the core of a cluster, three or four body dynamical encounters can produce exotic binaries whose formation is very unlikely, or impossible, in other Galactic environments: e.g. double millisecond pulsars, millisecond pulsar+black-hole, millisecond pulsar+intermediate mass black-hole.

Besides the completion of the GBGC, a new low-frequency drift scan survey is now ongoing at Green Bank radio telescope, with parameters $\Delta v=50 \mathrm{MHz} @ v=0.35 \mathrm{GHz}, 2048$ freq channels, $\Delta t=2.5 \mathrm{~min}, \delta t=81.92 \mu \mathrm{s}$. The first discovery of this experiment occurred on Sept 2007 [Lorimer, private comm]. A survey of the Galactic disk at intermediate frequency is also running using $\sim 20$ antennae at Giant Meter wave Radio Telescope, in India: $\Delta v=16 \mathrm{MHz} @ v=0.61$ $\mathrm{GHz}, 256$ freq channels, $\Delta t=35 \mathrm{~min}, \delta t=256 \mu \mathrm{s}$. Three pulsars have been already discovered during 2007 [McLaughlin, private comm]. At Parkes the PA-Survey (looking at the Galactic plane in the direction of the Perseus Arm) is about to be completed. The parameters are as for the PMSurvey, but with $\delta t=125 \mu \mathrm{s}$. Ten new pulsars have been detected to date [Burgay \& Keith, private comm]. However, the most important ongoing experiment is the P-ALFA survey of the Galactic disk at Arecibo [13], using a 7 beam receiver and the following parameters: $\Delta v=100 \mathrm{MHz}$ (to be upgraded to $300 \mathrm{MHz}$ ) $@ v=1.4 \mathrm{GHz}, \delta v=0.04 \mathrm{MHz}, \Delta t=67 \mathrm{~s}$ (or $134 \mathrm{~s}$ ), $\delta t=64 \mu \mathrm{s}$. This experiment has already produced some tens of discoveries: among them an interesting relativistic binary (PSR J1906+0746 [14]) and the first fully recycled pulsar in an eccentric orbit (and not belonging to a GC). In the end, many hundreds of new pulsars are expected from this survey.

The installation of a digital filter-bank for the 13 beam Parkes receiver at $1.4 \mathrm{GHz}$ will largely reduce dispersion smearing with respect to the analog filter-banks used so far. This will open the possibility of finding distant recycled pulsars in the Galaxy (and among them many relativistic 
binaries), as well as fast transients at high time resolution. A series of surveys with the new backend is then expected to begin since late 2008 [Bailes, Johnston, Kramer \& Possenti, private comm].

On a longer timescale, it is important to remember the exciting possibilities opened by SKA: this instrument will allow to detect a large fraction of the entire population of the not-recycled pulsars in the Galaxy (including many sources close to the Galactic center) and the first examples of pulsars residing in other galaxies. The sample of fast rotating recycled pulsars should also grow of about an order of magnitude with respect to now, with particular emphasis on the recycled pulsars located in ultra close binaries and/or having a very heavy (black-hole) companion: in fact, the discovery of these long sought systems will be extremely facilitated by the short integration times allowed by the huge instantaneous sensitivity of SKA. Observational and scientific perspectives of SKA in the field of pulsar study are discussed in full details e.g. in [15] and [16].

\section{References}

[1] R.N. Manchester, et al., The Parkes Southern Pulsar Survey. I. Observing and data analysis systems and initial results, MNRAS 279 (1235) 1996

[2] D.Lorimer and M. Kramer, Handbook of Pulsar Astronomy, Cambridge University Press 2005

[3] M. McLaughlin, et al., Transient radio bursts from rotating neutron stars, Nature 439 (817) 2006 [astro-ph/0511587]

[4] F. Camilo, et al., Transient pulsed radio emission from a magnetar, Nature, 442 (892) 2006 [astro-ph/0605429]

[5] R.N. Manchester, et al., The Parkes multi-beam pulsar survey - I. Observing and data analysis systems, discovery and timing of 100 pulsars, MNRAS, 328 (17) 2001 [astro-ph/0106522]

[6] R. Edwards, et al., The Swinburne intermediate-latitude pulsar survey, MNRAS, 326 (358) 2001 [astro-ph/0105126]

[7] M. Burgay, et al., The Parkes High-Latitude pulsar survey, MNRAS, 368 (283) 2006

[8] M. Burgay, et al., An increased estimate of the merger rate of double neutron stars from observations of a highly relativistic system, Nature 426 (531) 2003 [astro-ph/0312071]

[9] A.G. Lyne, et al., A Double-Pulsar System: A Rare Laboratory for Relativistic Gravity and Plasma Physics, Science 303 (1153) 2004 [astro-ph/0401086]

[10] S. Ransom, et al., Twenty-One Millisecond Pulsars in Terzan 5 Using the Green Bank Telescope, Science 307 (862) 2005 [astro-ph/0501230]

[11] J.W.T Hessel, et al., A Radio Pulsar Spinning at 716 Hz, Science 311 (1901) 2006 [astro-ph/0601337]

[12] P.C.C. Freire, et al., Eight New Millisecond Pulsars in NGC 6440 and NGC 6441, arXiv:0711.0925

[13] J.M., Cordes, et al, Arecibo Pulsar Survey Using ALFA. I. Survey Strategy and First Discoveries, ApJ, 636 (446) 2006 [astro-ph/0509732]

[14] D. Lorimer, et al., Arecibo Pulsar Survey Using ALFA. II. The Young, Highly Relativistic Binary Pulsar J1906+0746, ApJ 640 (428) 2006 [astro-ph/0511523]

[15] J.M., Cordes, et al, Pulsars as Tools for Fundamental Physics \& Astrophysics New Astronomy Reviews 48 (1413) 2004, [astro-ph/0505555]

[16] M. Kramer, et al, Strong-field tests of gravity using pulsars and black holes, New Astronomy Reviews 48 (993) 2004 [astro-ph/0409020] 